\title{
SOME PROPERTIES OF CERTAIN RELATIVELY FREE GROUPS
}

\section{T. C. HURLEY}

ABSTRACT. It is shown that the groups $F /\left[F_{n}, F_{m}\right]$, where $F$ is a free group and $m, n$ are positive integers such that $m<n \leq 2 m$, are residually "tor sion-free and nilpotent", and the structure of their lower central factors is computed.

Let $m, n$ be positive integers such that $m<n \leq 2 m$. The groups $\left.F / F_{n}, F_{m}\right]$, where $F$ is a free group, are studied. It is shown that $\left[F_{n-1}, F_{m}\right] /\left[F_{n}, F_{m}\right]$ is a free abelian group and a basis is given. The structure of the lower central factors of $F /\left[F_{n}, F_{m}\right]$ is computed, and it is shown that they are free abelian groups. Then, by showing it is residually nilpotent, it is deduced that $F /\left[F_{n}, F_{m}\right]$ is residually "torsion-free and nilpotent" and, hence, that it is residually a finite $p$-group for all primes $p$.

If $a$ and $b$ are elements of a group $G$, then the commutator $[a, b]=$ $a^{-1} b^{-1} a b$. Higher commutators are left-normed. If $H$ and $K$ are subsets of $G$, then $[H, K]$ is the subgroup of $G$ generated by all $[h, k]$ with $h$ in $H$ and $k$ in $K$. The lower central series of $G$ is defined by $G_{1}=G, G_{n+1}=$ $\left[G_{n}, G\right]$. The $n$th lower central factor of $G$ is $G_{n} / G_{n+1}$. Throughout, $F$ is a free group of at most countable rank, freely generated by $X=\left\{x_{1}, x_{2}, \ldots\right\}$. The reader is referred to Marshall Hall [4, pp. 165-175] for the definition and properties of basic commutators. Note that the basic commutators are ordered according to weight, and a definite ordering is fixed on the basic commutators of the same weight. If $a$ and $b$ are basic commutators then $a \geq b(a>b)$ means that $a$ is greater than or equal to $b$ (respectively, greater than but not equal to $b$ ) in the ordering of the basic commutators. Assume throughout that $m$ and $n$ are positive integers with $m \leq n \leq 2 m$; for Lemmas $2,3,4,5$ and Theorem 6 it is further assumed that $m<n$.

Received by the editors December 12, 1973.

AMS (MOS) subject classifications (1970). Primary 20E25, $20 \mathrm{~F} 35$.

Key words and phrases. Relatively free group, residual property, lower central factors. 
The result

$$
\left[G_{i}, G_{j}\right] \subseteq G_{i+j} \quad[4, \text { Corollary 10.3.5] }
$$

will be used frequently without further reference. A group $G$ is said to be "residually P", where $\mathrm{P}$ is some property of groups, if, for every $g \neq 1$ in $G$, there exists a normal subgroup $N_{g}$ of $G$ such that $G / N_{g}$ has the property $\mathrm{P}$ (see $[5, \mathrm{pp} .27-32])$.

Lemma 1. If $x \in G_{k}, y \in G_{p}$ and $z \in G_{l}$ then

$$
\begin{aligned}
{[x, y, z] \equiv } & {[y, z, x]^{-1}[x, z, y] \text { modulo }\left[\left[G_{k}, G_{p}\right],\left[G_{l}, G_{p}\right]\right] } \\
& \times\left[\left[G_{k}, G_{l}\right],\left[G_{l}, G_{p}\right]\right] \times\left[\left[G_{k}, G_{l}\right],\left[G_{k}, G_{p}\right]\right] .
\end{aligned}
$$

Proof. By the commutator identities in [4, p. 150],

$$
\begin{aligned}
& {[x, y z]=[x, z][x, y][x, y, z]=[x, z y[y, z]] } \\
&= {[x,[y, z]][x, z y][x, z y,[y, z]] } \\
&= {[x,[y, z]][x, y][x, z][x, z, y][[x, y][x, z][x, y, z],[y, z]] } \\
& \equiv {[x,[y, z]][x, y][x, z][x, z, y] } \\
& m \text { odulo }\left[\left[G_{k}, G_{p}\right],\left[G_{l}, G_{p}\right]\right] \times\left[\left[G_{k}, G_{l}\right],\left[G_{l}, G_{p}\right]\right] \\
& \equiv {[x, z][x, y][y, z, x]-1[x, z, y] } \\
& \operatorname{modulo}\left[\left[G_{k}, G_{l}\right],\left[G_{k}, G_{p}\right]\right] \times\left[\left[G_{k}, G_{p}\right],\left[G_{l}, G_{p}\right]\right] \times\left[\left[G_{k}, G_{l}\right],\left[G_{l}, G_{p}\right]\right]
\end{aligned}
$$

The result now follows.

The following lemma is easily deduced from the commutator identities.

Lemma 2. If $a, b \in G_{n-1}, c, d \in G_{m}$, then

$$
[a b, c d] \equiv[a, c][a, d][b, c][b, d] \operatorname{modulo}\left[G_{n}, G_{m}\right] .
$$

If, further, $b \in G_{n}$, then

$$
[a b, c d] \equiv[a, c][a, d] \operatorname{modulo}\left[G_{n}, G_{m}\right],
$$

and if $d \in G_{n}$ then

$$
[a b, c d] \equiv[a, c][b, c] \text { modulo }\left[G_{n}, G_{m}\right] .
$$

Also

$$
\left[a^{-1}, c\right] \equiv[a, c]^{-1} \equiv\left[a, c^{-1}\right] \text { modulo }\left[G_{n}, G_{m}\right] .
$$

Lemma 3. Let $a$ be a basic commutator of weight $t$ such that $a=$ $\left[a_{1}, c\right]$, where $a_{1}$ and $c$ are basic commutators of weights $t-q$ and $q$, 
respectively, and let $b$ be a basic commutator of weight $p$ such that $a>b$ and such that $p \leq t<3 p$. Then either $[a, b]$ is a basic commutator or else

$$
[a, b] \equiv\left[c, b, a_{1}\right]^{-1}\left[a_{1}, b, c\right] \text { modulo }\left[F_{t+1}, F_{p}\right]
$$

where $\left[c, b, a_{1}\right]$ and $\left[a_{1}, b, c\right]$ are basic commutators.

Proof. $[a, b]$ is a basic commutator if $c \leq b$. If $c>b$, then weight $c$ $\geq p$, and thus $2 p>$ weight $a_{1} \geq$ weight $c$; this implies that $\left[c, b, a_{1}\right]$ and $\left[a_{1}, b, c\right]$ are basic commutators, and by Lemma 1 ,

$$
\begin{aligned}
{\left[a_{1}, c, b\right] \equiv } & {\left[c, b, a_{1}\right]^{-1}\left[a_{1}, b, c\right] \text { modulo }\left[\left[F_{t-q}, F_{q}\right],\left[F_{p}, F_{q}\right]\right] } \\
& \times\left[\left[F_{t-q}, F_{p}\right],\left[F_{p}, F_{q}\right]\right] \times\left[\left[F_{t-q}, F_{p}\right],\left[F_{t-q}, F_{q}\right]\right] .
\end{aligned}
$$

By the three subgroup lemma [4, Theorem 10.3.5, p. 155], the group on the right side of the above equation is contained in $\left[F_{t+1}, F_{p}\right]$. (Note that $2 p>t-q \geq q \geq p$ and, hence, $\left.F_{t-q} \subseteq F_{q} \subseteq F_{p}\right)$

Set

$$
F(n+m+k)=\prod_{i+j=k}\left[F_{n+i}, F_{m+j}\right]
$$

and

$S=\{[x, y] / x$ a basic commutat or of weight $n-1$ and

$y$ a basic commutator of weight $t, m \leq t \leq n-1\}$,

and for each $k, k=-1,0, \cdots, n-m-2$, let

$$
\begin{aligned}
& S_{k}=\{[x, y] / x \text { a basic commutator of weight } n-1 \text { and } \\
& \qquad y \text { a basic commutator of weight } m+k+1\} .
\end{aligned}
$$

Then $S=\bigcup_{i=-1}^{n-m-2} S_{i}$, where the union is disjoint.

Also define, for $k=0,1, \cdots, n-m-2$,

$T_{k}=\{[x, y] / x$ a basic commutator of weight $n+i, y$ a basic

commutat or weight $m+j$, with $i+j=k\}$.

Lemma 4. $\left[F_{n-1}, F_{m}\right] /\left[F_{n}, F_{m}\right]$ is generated by $S$.

Proof. $\left[F_{n-1}, F_{m}\right]$ is generated by $[a, b], a \in F_{n-1}, b \in F_{m}$. Now $a$ is congruent to a product of basic commutators of weight $n-1$ modulo $F_{n}$, and $b$ is congruent to a product of basic commutators of weights $m, m+1$, $\cdots, n-1$ modulo $F_{n}$. Applying Lemma 2 now gives the required result.

Lemma 5. $F(n+m+k) / F(n+m+k+1)$ is generated by the elements of $T_{k}$ that are basic $(0 \leq k \leq n-m-2)$. 
Proof. It is clear that $F(n+m+k) / F(n+m+k+1)$ is generated by $T_{k}$. If $[a, b] \in T_{k}$ and $[a, b]$ is not a basic commutator, then $a=\left[a_{1}, c\right]$, where $c>b$. Let weight $a=n+i$, weight $b=m+j$, and weight $c=q$ $(i+j=k)$.

Then by Lemma 3,

$$
\left[a_{1}, c, b\right] \equiv\left[c, b, a_{1}\right]^{-1}\left[a_{1}, b, c\right] \text { modulo }\left[F_{n+i+1}, F_{m+j}\right] \text {, }
$$

where $\left[c, b, a_{1}\right]$ and $\left[a_{1}, b, c\right]$ are basic commutators.

Now weight $a_{1} \geq$ weight $c \geq$ weight $b \geq m$ and $2 m \geq n$. Hence $\left[c, b, a_{1}\right]$ and $\left[a_{1}, b, c\right]$ are contained in $T_{k}$; this completes the proof.

Corollary. $\left[F_{n}, F_{m}\right]$ is generated, modulo $F(n+m+k+1)$, by the elements of $T_{0} \cup T_{1} \cup \cdots \cup T_{k}$ that are basic $(0 \leq k \leq n-m-2)$.

We are now in a position to prove

Theorem 6. $\left[F_{n-1}, F_{m}\right] /\left[F_{n}, F_{m}\right]$ is freely generated as an abelian group by $S$.

Proof. This follows directly from the basis theorem [4, Theorem 11.2.4, p. 175], the Corollary to Lemma 5, the fact that the elements of $S$ are basic commutators, that $S_{k} \cap T_{k}=\varnothing$ (null set), and that $F(n+m+k) \subseteq F_{n+m+k}$.

The $l$ th lower central factor of $F /\left[F_{n}, F_{m}\right]$ is $F_{l}\left[F_{n}, F_{m}\right] / F_{l+1}\left[F_{n}, F_{m}\right]$. It is clear that this group (call it $G(n, m, l)$ ) is generated by

$R(n, m, l)=\{x / x$ a basic commutator of weight $l$ and if $x=[y, z]$ where $y$ and $z$ are basic commutators with weight $z \geq m$, then weight $y<n\}$.

It is known (Šmel'kin [3]) that $G(m, m, l)$ is free abelian with basis $R(m, m, l)$ and we prove here

Theorem 7. $G(n, m, l)$ is free abelian with basis $R(n, m, l)$.

Proof. We proceed by induction on $n-m$. The case $n-m=0$ follows by the above-mentioned result of Šmel'kin. Assume $G(n-1, m, l)$ is generated freely by $R(n-1, m, l)$. By taking the natural map $\phi: G(n, m, l) \rightarrow$ $G(n-1, m, l)$, we see that it suffices to prove that the following set $Q$ is independent modulo $F_{l+1}\left[F_{n}, F_{m}\right]$ :

$Q=\{x / x$ a basic commutator of weight $l$ where $x=[y, z]$,

$y$ a basic commutator of weight $n-1$ and

$z$ a basic commutator of weight $l-(n-1) \geq m\}$. 
This follows at once from the Corollary to Lemma 5-note that $Q=\varnothing$ if $l \geq 2 n-1$.

Theorem 8. $F /\left[F_{n}, F_{m}\right]$ is residually nilpotent.

Proof. The proof is by induction on $n-m$. When $n-m=0$ the result follows from Gruenberg [1]. Suppose $n-m>0$ and let $g \in F, g \notin\left[F_{n}, F_{m}\right]$. Then we need to find $N_{g}$ with $\left[F_{n}, F_{m}\right] \subseteq N_{g} \triangleleft F$ (where $\triangleleft$ means "normal in') such that $F / N_{g}$ is nilpotent and $g \notin N_{g}$. If $g \notin\left[F_{n-1}, F_{m}\right]$, then by the inductive hypothesis there exists $N_{g}$ with $\left[F_{n-1}, F_{m}\right] \subseteq N_{g} \triangleleft F$ such that $F / N_{g}$ is nilpotent and $g \notin N_{g}$, and this $N_{g}$ will suffice in this situation.

If $g \in\left[F_{n-1}, F_{m}\right]\left(g \notin\left[F_{n}, F_{m}\right]\right)$, then $g$ is a nontrivial product of elements of $S$ (which are basic) modulo $\left[F_{n}, F_{m}\right]$. By corollary to Lemma 5, $\left[F_{n}, F_{m}\right]$ is generated modulo $F_{2 n-1}$ by the elements of $T_{0} \cup T_{1} \cup \ldots$ $\cup T_{n-m-2}$ that are basic, and since $S \cap\left(T_{0} \cup T_{1} \cup \cdots \cup T_{n-m-2}\right)=\varnothing$, it follows that $g \notin F_{2 n-1}\left[F_{n}, F_{m}\right]$. Thus $\left[F_{n}, F_{m}\right] \subseteq F_{2 n-1}\left[F_{n}, F_{m}\right] \triangleleft F$, $F / F_{2 n-1}\left[F_{n}, F_{m}\right]$ is nilpotent and $g \notin F_{2 n-1}\left[F_{n}, F_{m}\right]$. Hence $N_{g}=$ $F_{2 n-1}\left[F_{n}, F_{m}\right]$ suffices in this situation.

Theorem 9. $F /\left[F_{n}, F_{m}\right]$ is residually "torsion-free and nilpotent".

Proof. This follows at once from Theorems 7 and 8.

Corollary. $F /\left[F_{n}, F_{m}\right]$ is residually a finite $p$-group for all primes $p$.

Proof. This need only be proved when $F$ is finitely generated. By Gruenberg [1] a finitely-generated torsion-free nilpotent group is residually a finite $p$-group for all primes $p$ and a residually "residually a finite $p$-group" is residually a finite $p$-group.

Since $F /\left[F_{n}, F_{m}\right]$ has torsion-free lower central factors (Theorem 7 ), it follows from a result of P. M. Cohn [2] that the so-called dimension subgroups and the lower central series of $F /\left[F_{n}, F_{m}\right]$ coincide.

\section{REFERENCES}

1. K. W. Gruenberg, Residual properties of infinite soluble groups, Proc. London Math. Soc. (3) 7 (1957), 29-62. MR 19, 386.

2. P. M. Cohn, Generalisation of a theorem of Magnus, Proc. London Math. Soc. (3) 2 (1952), 297-310; correction, ibid. MR 14, 532.

3. A. L. Šmel'kin, Free polynilpotent groups, Izv. Akad. Nauk SSSR Ser. Mat. 28 (1964), 91-122; English transl., Amer. Math. Soc. Transl. (2) 55 (1966), 270304. MR $29 \# 161$. 
4. Marshall Hall, The theory of groups, Macmillan, New York, 1959. MR 21 \#1996.

5. Hanna Neumann, Varieties of groups, Ergebnisse der Mathematik und ihrer Grenzgebiete, Band 37, Springer-Verlag, Berlin and New York, 1967. MR 35 \#6734.

DEPARTMENT OF PURE MATHEMATICS, THE UNIVERSITY, SHEFFIELD S3 7RH, ENGL AND

Current address: Department of Mathematics, University College, Belfield, Dublin 4, Ireland 